Quantitative real-time polymerase chain reaction: NK cells were sorted from using isolation kit from stemcell ${ }^{\mathrm{TM}}$ (cat no.17955). cDNA was synthesized from RNA separated from isolated nk cells. Gene primers were run on the StepOnePlus RealTime PCR Systems and analyzed with StepOne Software V2.1 (Applied Biosystems New York, USA).

Results Fraction of cd3-cd56 in pbmc (\%) were reduced in SLE patients $(5.395 \% \pm 0.6900 \mathrm{~N}=27)$ as compared to healthy control $(10.32 \pm 1.729 \mathrm{~N}=16)$, P Value0.0037

1. ROS increased in $\mathrm{cd} 3-\mathrm{cd} 56+$ cells of SLE patients as compared to healthy control.

2. Cd56 dim and cd56 bright cells of SLE have significantly higher fraction expressing kir2dl4.

3. Lower Cd56 dim cells of SLE patients express kir3dl1 on surface.

4. Mean mRNA level in SLE patients of HMOX-1 is 2.56 times higher as compared to healthy control. $(\mathrm{p}-\mathrm{value}=0.0532)$

5. Mean mRNA Expression level of kir3dl1 are not significantly different in SLE patients and healthy control

6. Mean mRNA expression level of kir2dl4 is 20 times higher in SLE patients as compared to healthy control(pvalue $=0.0260$ )

Conclusion In our study NK cell of patients have been found more oxidatively stressed as compared to healthy and Increased mRNA expression of HMOX-1 also indicate same. Significantly increased expression of KIR2dl4 at mRNA and surface expression may be responsible for killing HLA-G bearing self cells. Surface expression of Kir3dl1 has been found to be significantly decreased in CD 56 dim cells, probable consequence is reduced tolerance. Although we could find any significant correlation of mRNA expression kir2dl4 and kir3dl1 with that of hmox-1, so cannot conclude any relationship between these receptor and oxidative stress.

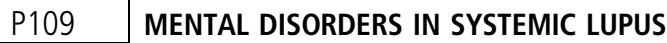 ERYTHEMATOSUS AND ANTIPHOSPHOLIPID SYNDROME PATIENTS}

${ }^{2}$ Anastasia Borisova, ${ }^{1}$ Fariza Cheldieva, ${ }^{1}$ Tatiana Lisitsyna, ${ }^{1}$ Tatiana Reshetnyak, ${ }^{2}$ Dmitry Veltishchev, ${ }^{2}$ Olga Seravina, ${ }^{2}$ Oksana Kovalevskaya. ${ }^{1}$ Research Institute of Rheumatology named after V.A. Nasonova, Moscow; ${ }^{2}$ Moscow Research Institute of Psychiatry, Serbsky NMRC PN MoH, Moscow, Russian Federation

\subsection{6/lupus-2020-eurolupus. 153}

Background Mental disorders (MD) in systemic lupus erythematosus (SLE) and antiphospholipid syndrome (APS) patients has been poorly described and recognized.

Objective To describe the frequency and spectrum of mental disorders in SLE and APS patients.

Methods 71 patients with mean $(\mathrm{M} \pm \mathrm{SD})$ age $38,8 \pm 11,7$ years were enrolled in the study: 21 of proven SLE patients, 26 SLE with secondary APS and 24 - with proven primary APS (PAPS). $54(76,1 \%)$ patients were women. SLE activity was measured by SLEDAI scale. MD were diagnosed by psychiatrist in accordance with the Hospital Anxiety and Depression Scale (HADS) and ICD-10 in semi-structured interview. The severity of depression and anxiety was evaluated by Montgomery-Asberg Depression Rating Scale (MADRS) and Hamilton Anxiety Rating Scale (HAM-A). CD were diagnosed with psychology and neuropsychology methods.
Abstract P109 Table 1 Prevalence of mental and cognitive disorders in SLE, SLE+APS and PAPS patients

\begin{tabular}{|c|c|c|c|c|}
\hline \multirow[t]{2}{*}{ Sample characteristics } & SLE, $n=21$ & $\begin{array}{l}\text { SLE+APS, } \\
n=26\end{array}$ & $\begin{array}{l}\text { PAPS, } \\
n=24\end{array}$ & $\mathbf{P}$ \\
\hline & 1 & 2 & 3 & \\
\hline \multirow[t]{3}{*}{ Gender (malelfemale), n (\%) } & 0121 & 6120 & $11 \backslash 13$ & Ns \\
\hline & $(0 \% \backslash 100 \%)$ & $(23,1 \%$ & $(45,8 \%$ & \\
\hline & & $176,9 \%)$ & $154,2 \%)$ & \\
\hline Age, years & $36,9 \pm 14,7$ & $39,3 \pm 10,6$ & $39,8 \pm 10,2$ & Ns \\
\hline SLEDAl, point & $6,4 \pm 5,7$ & $6,8 \pm 5,4$ & - & ns \\
\hline \multicolumn{5}{|l|}{ Mental disorders, n (\%) } \\
\hline Anxiety-depressive disorders: & $20(95,2 \%)$ & $18(69,2 \%)$ & $19(79,2 \%)$ & $P_{1-2}=0,03$ \\
\hline - recurrent depressive disorder & $10(47,6 \%)$ & $9(34,6 \%)$ & $10(41,7 \%)$ & ns ns ns ns \\
\hline - single depressive episode & $2(9,5 \%)$ & - & $1(4,2 \%)$ & \\
\hline - dysthymia & $6(28,6 \%)$ & $9(34,6 \%)$ & $6(25,0 \%)$ & \\
\hline - generalized anxiety disorder & $2(9,5 \%)$ & - & $2(8,3 \%)$ & \\
\hline Bipolar disorder & $1(4,8 \%)$ & $2(7,7 \%)$ & - & ns \\
\hline Schizotypal disorder & $2(9,5 \%)$ & $2(7,7 \%)$ & $1(4,2 \%)$ & ns \\
\hline Vascular dementia & - & $2(7,7 \%)$ & $1(4,2 \%)$ & ns \\
\hline Epileptic syndrome & $1(4,8 \%)$ & $6(23,1 \%)$ & $3(12,5 \%)$ & ns \\
\hline \multicolumn{5}{|l|}{ Cognitive impairment, $\mathbf{n}(\%)$} \\
\hline \multicolumn{5}{|l|}{ impairment } \\
\hline severe cognitive impairment & $4(19,0 \%)$ & $5(19,2 \%)$ & $2(8,3 \%)$ & ns \\
\hline
\end{tabular}

Results The patients with SLE, SLE+APS and PAPS didn't differ in age, but in PAPS group men met more often. The groups didn't differ in SLE activity. The rate of MD (mainly anxiety-depressive disorders) were high, highest in SLE patients. Recurrent depressive disorder met more often. The prevalence of anxiety-depressive disorders: recurrent depressive disorder, single depressive disorder, dysthymia, generalized anxiety disorder was significantly higher in SLE than in SLE+APS groups $(p=0,03)$. Bipolar disorder didn't meet in PAPS patients. Epileptic syndrome met more often (ns) in SLE+APS patients. The prevalence of schizotypal disorder was higher (ns) in patients with SLE (SLE and SLE+APS groups) (also higher than in population). Vascular dementia was diagnosed more often (ns) in APS patients (SLE+APS and PAPS groups). The rate of cognitive difficulties was very high in all groups, with predominance of mild and moderate impairment.

Conclusion Chronic depressive and cognitive disorders are typical for patients with SLE, SLE+APS and PAPS. Mild and moderate cognitive disorders diagnosed in PAPS-patients significantly more often $(\mathrm{p}<0,05)$ than in SLE patients without APS.

\section{P110 LUPUS EUROPE - EXERCISE PROGRAM FOR LUPUS PATIENTS}

${ }^{1,2,3}$ Jeanette Andersen, ${ }^{1}$ Alain Cornet, ${ }^{1}$ Kirsi Myllys, ${ }^{1}$ Anne Charlet, ${ }^{1}$ Annemarie Sluijmers, ${ }^{1}$ Helga Ovens. 'Lupus Europe, Essex, UK; ${ }^{2}$ Lupus DK, Denmark; ${ }^{3}$ EULAR PARE Board, Zurich, Switzerland

\subsection{6/lupus-2020-eurolupus.154}

Background Up to $80 \%$ of all Lupus patients experience fatigue and most of them report this as the most severe symptom. One of the major causes of morbidity in SLE patients is 